\title{
INTERVENCIÓN GRUPAL ONLINE PARA LA ELABORACIÓN DEL DUELO Y RECUPERACIÓN DEL BIENESTAR PSICOLÓGICO TRAS UNA RUPTURA AMOROSA
}

\section{Grief work and psychological wellbeing after a romantic breakup: an online group intervention.}

\section{Perla Navarro Vásquez \\ Psicóloga miembro del grupo de profesionales de esta especialidad psicológicamente. \\ Correo-e: perlannv@gmail.com, https://orcid.org/0000-0001-5308-0962}

Cómo citar: Navarro Vásquez, P. (2020). Intervención grupal online para la elaboración del duelo y recuperación del bienestar psicológico tras una ruptura amorosa. Ciencia y Sociedad, 45(4), 119-132. Doi: https://doi.org/10.22206/cys.2020.v45i4.pp119-132

\begin{abstract}
Resumen
Este estudio buscó verificar si aumentaban los niveles de bienestar psicológico en personas que han vivido una ruptura amorosa, luego de participar de una intervención grupal de modalidad online basada en el modelo de tareas del duelo de Worden, y el uso de técnicas recomendadas por este autor para el trabajo de duelo. La muestra final del estudio estuvo compuesta por 12 participantes adultos, residentes en la República Dominicana. La recolección de datos cuantitativos se hizo con el uso de las Escalas de Bienestar Psicológico de Ryff en su adaptación española realizada por Rodríguez-Carvajal et al. (2010). Además, se recolectaron datos cualitativos a través de un cuestionario de preguntas abiertas. Los resultados muestran un aumento significativo en los niveles de bienestar psicológico tras la intervención ( $m=28.58 ; t=5.86, p<0.001)$. Los hallazgos cualitativos, clasificados en cinco categorías principales, evidencian la percepción de cambios positivos en los participantes luego de haber participado de la intervención, como el desarrollo de la empatía con los miembros del grupo, el cambio de ideas erróneas que pudieran interferir en el proceso de duelo y la motivación para continuar procesos terapéuticos. Se recomienda realizar otras investigaciones con una muestra mayor y con grupo control, de modo que puedan hacerse generalizaciones y conclusiones definitivas sobre la efectividad del programa diseñado y aplicado.
\end{abstract}

Palabras clave: divorcio; psicología; grupo; bienestar.

\begin{abstract}
This study sought to verify if the levels of psychological well-being increased in people who had experienced a romantic breakup, after participating in an online group intervention based on Worden's grief tasks model, and the use of techniques recommended by this author for grief work. The final sample of the study consisted of 12 adult participants, residents of the Dominican Republic. The quantitative data collection was done with the use of the Ryff Psychological Well-being Scales in its Spanish adaptation carried out by Rodríguez-Carvajal et al. (2010). In addition, qualitative data were collected through an open-ended questionnaire. The results show a significant increase in the levels of psychological well-being after the intervention $(\mathrm{m}=28.58 ; \mathrm{t}=5.86, \mathrm{p}<0.001)$. The qualitative findings, classified into five main categories, show the perception of positive changes in the participants, such as the development of empathy with the group members, the change of erroneous ideas that could interfere in the grief process and motivation to continue therapeutic processes. It is recommended to carry out other investigations with a larger sample and with a control group, so that generalizations and definitive conclusions can be made about the effectiveness of the designed and applied program.
\end{abstract}

Keywords: divorce; psychology; group; wellbeing. 


\section{Introducción}

La ruptura de una relación amorosa es una experiencia de pérdida que las personas pueden experimentar una o varias veces en su vida. Diversos autores plantean que, al igual que sucede con otras pérdidas, esta experiencia genera un proceso de duelo (Morales, 2019; Yárnoz-Yaben, 2017; Sierra, Rodríguez \& Torres-Torija, 2017; Raya, Sierra \& Velasco, 2013; Cuervo, Moreno \& Concha, 2009; Testor, Pujol, Vidal \& Alegret, 2009).

La pérdida de una relación de pareja tiene un impacto negativo en el sentido de bienestar de la persona. Generalmente se ha estudiado este impacto con relación al divorcio, pero estas consecuencias también son vividas por las personas que pasan por la ruptura de una pareja que, aun no habiendo formalizado un matrimonio, desarrollan una relación estable y que consideran seria (Sierra et al., 2017; Yárnoz-Yaben \& González, 2012).

Tras una ruptura amorosa, como sucede en otras experiencias de duelo, pueden presentarse síntomas físicos, emocionales, cognitivos y conductuales. A nivel físico, pueden aparecer alteraciones en los patrones de sueño, hipersensibilidad, fatiga, debilidad muscular, opresión de pecho y garganta, falta de aire y palpitaciones; a nivel emocional, sentimientos de hostilidad y rencor, sensación de soledad y desamparo, episodios de angustia por la separación, sensación de alivio, tristeza profunda, culpa, ansiedad, enojo, impotencia, nostalgia, sensación de inadecuación; en el ámbito cognitivo, son comunes síntomas como la confusión, duda por haber fallado, ideas de fracaso, ideas de incapacidad, daños en la autoestima, incredulidad, pensamientos rumiantes, dificultad para la concentración, y problemas de memoria; en el ámbito conductual, se puede observar el atesoramiento de objetos, llanto, aislamiento, conductas autodestructivas, evitación de lugares y actividades que traigan recuerdos de la ex pareja, buscar a la ex pareja, entre otros. Todos estos síntomas han sido obser- vados como comunes en procesos de duelo normal, sin embargo, no son un cuadro fijo. Al igual que en cualquier proceso de duelo, en las rupturas de pareja, esta experiencia es vivida por cada persona de manera distinta (Worden, 2018; Sierra et al., 2017; Yárnoz-Yaben, 2017; Bucio, 2007 citado por Raya et al., 2013; Muñiz Tinajero, 2010 citado por Aragón y Cruz, 2014; Cuervo et al., 2009; Testor et al., 2009; Fernández- Liria, Rodríguez-Vega $\&$ Diéguez-Porres, 2006).

El impacto negativo que tiene la ruptura en el bienestar psicológico se hace más evidente cuando la persona tiene dificultades para aceptar la separación, no logra desvincularse totalmente y trata de mantener un lazo, deteniendo así el proceso de elaboración del duelo. Cuando la persona elabora de manera adecuada el duelo, logra hacer un cierre y avanza hacia la adaptación, permitiendo una recuperación del sentido de bienestar y satisfacción, así como la capacidad para el manejo de conflictos y el perdón (Yárnoz-Yaben, 2017 y Doménech, 1994 citados por Sierra et al., 2017; Márquez, 2005 citado por Raya et al., 2013).Uno de los enfoques desde los cuales se ha explicado cómo sucede la elaboración del duelo o procesamiento de la pérdida, es el planteado por Worden, quien propone una serie de tareas que la persona debe realizar para lograr una adaptación a la vida sin el objeto perdido. Desde este enfoque el duelo se trata de un proceso, no de un estado; es una labor en la que la persona está activamente implicada (Worden, 2018).

Las cuatro tareas que integran el procesamiento del duelo propuestas por Worden (2018) son:

1) Aceptar la realidad de la pérdida, que se refiere a reconocer la cualidad definitiva e irreversible de la pérdida.

2) Vivir el dolor de la pérdida, que implica conectar con los sentimientos que provoca la pérdida. 
3) Adaptarse a un mundo sin el objeto perdido, que se refiere a que la persona haga ajustes a su vida, como la adquisición de habilidades y asunción de roles que anteriormente pertenecían a la otra persona, así como la reparación de su autoconcepto.

4) Recolocar lo perdido de modo que pueda dirigirse la energía emocional y vital hacia otras relaciones y hacia la vida.

Muchas personas son capaces de afrontar este proceso por sí mismas, adaptándose satisfactoriamente a la vida luego de la pérdida. Sin embargo, algunos individuos experimentan dificultades significativas en este proceso y buscan asistencia profesional para el procesamiento del duelo porque perciben que puede ser de ayuda. En estos casos, la intervención en trabajo de duelo puede facilitar una adaptación más efectiva a la pérdida (Schut, 2010, citado por Worden, 2018; Cuervo et al., 2009).

Algunos estudios han demostrado la eficacia de intervenciones grupales con diversos enfoques para el trabajo del duelo por ruptura amorosa o divorcio en adultos. Se encontraron evidencias de una disminución de la sintomatología ansiosa y depresiva, promoción de una mayor habilidad para vivir en el presente, formar relaciones significativas y mayor independencia, así como una valoración positiva de los participantes con relación a la expresión de sus sentimientos, adaptación y la comprensión del proceso de duelo (Guillén, Montaño \& Gordillo; 2015; Lee \& Hett, 1990). También se han encontrado estudios con diferentes poblaciones, que demuestran que los participantes de intervenciones grupales se beneficiaron significativamente en términos del ajuste post divorcio y en la facilitación del duelo por divorcio. (Quinney \& Fouts, 2008 Vukalovich \& Caltabiano, 2008; Raya et al., 2013; Najjari, Khodabakhshi Koolaee \& Falsafinejad, 2017; Nameni, Mohammadipoor \& Noori, 2017; Saadati, Rostami \& Darbani, 2017).
Asimismo, varias investigaciones, con poblaciones distintas, han demostrado la eficacia de intervenciones a través del internet para el trabajo del duelo por distintas pérdidas en adultos, encontrando reducción de sintomatología postraumática y resultados positivos significativos con relación a la salud mental en general (Fiegelman, Gorman, Beal \& Jordan, 2008 citados por Worden, 2018; Elder \& Burke, 2015; Kersting, et al., 2013; Kersting, Kroker, Schlicht, Baust, \& Wagner, 2011; Wagner \& Maercker, 2008; Wagner, Knaevelsrud, \& Maercker, 2007).

En este sentido, el objetivo de esta investigación fue desarrollar una intervención de modalidad virtual basada en la facilitación de las tareas del duelo de Worden para personas que habían vivido una ruptura amorosa, y verificar si luego de la intervención mostraban mejoras en su bienestar psicológico. El programa estuvo basado en actividades psicoeducativas sobre las tareas del duelo, ejercicios de enfoque cognitivo conductual como la identificación de pensamientos y creencias y la reestructuración cognitiva, así como técnicas sugeridas por Worden (2018) para la facilitación de las tareas del duelo, como: construcción de narrativas, uso de símbolos, creación de dibujos, uso de lenguaje evocativo, y el uso de metáforas.

\section{Método}

\section{Diseño metodológico}

Este es un estudio de metodología mixta cuasi experimental, con un diseño anidado concurrente de modelo cuantitativo dominante. El diseño anidado concurrente recolecta datos de carácter cualitativo y cuantitativo de manera simultánea. Los datos recolectados con el método de menor dominancia son anidados dentro del método que se considera central, y ambos resultados son comparados o triangulados en la fase de análisis (Sampieri, Collado \& Lucio, 2010). 


\section{Participantes}

La muestra inicial para este estudio estuvo compuesta por 20 participantes, adultos, mayores de 18 años, dominicanos, que habían vivido una ruptura amorosa o divorcio, ocurrida en un periodo no menor a seis semanas. Los mismos fueron reclutados a través de un muestreo no probabilístico de selección a conveniencia, a través de publicaciones de convocatoria utilizando distintas plataformas de redes sociales en línea. Para la selección se tomaron en cuenta los siguientes criterios de inclusión y exclusión:

\section{Inclusión:}

- Ser mayor de 18 años.

- Haber terminado una relación amorosa que consideran seria.

- Exclusión:

- Presencia de sintomatología depresiva severa o presencia de ideación suicida. Se aplicó el Inventario de Depresión de Zung, instrumento de libre acceso.

- Ruptura con menos de seis semanas de haber ocurrido.

De esta muestra inicial, solo 18 participantes completaron el proceso de selección. Dos personas completaron el instrumento de medición, pero no concluyeron la etapa de entrevista, y dos sí la completaron, pero fueron referidas a otros servicios de asistencia psicológica por presentar signos de depresión mayor. Se inició entonces la intervención con 16 participantes, de los cuales cuatro se retiraron del estudio. Por tanto, la muestra final fue de 12 participantes, con edades entre $19 \mathrm{y}$ 54 años, siendo la edad promedio de 29.08 años $(D E=10.75)$. De estos, 11 eran mujeres y un participante era hombre.

\section{Instrumentos}

Se aplicaron las Escalas de Bienestar Psicológico de Ryff, en su adaptación española realizada por Rodríguez-Carvajal et al., (2010). El instrumento cuenta con ocho escalas tipo Likert que van del 1 (totalmente en desacuerdo) al 6 (totalmente de acuerdo) que son: autonomía, autoaceptación, relaciones positivas, dominio del entorno, propósito en la vida, crecimiento personal, vitalidad y recursos internos. El nivel de confiabilidad obtenido fue de $\alpha=0.83$.

Además, se elaboró una entrevista semiestructurada de 12 preguntas para identificar las distintas maneras en que los participantes percibían que la ruptura amorosa había tenido impacto en las esferas de su vida y los cambios percibidos luego de la intervención.

\section{Procedimiento}

Los participantes recibieron un documento digital que contenía el consentimiento informado y las Escalas de Bienestar Psicológico (Rodríguez-Carvajal et al., 2010), las cuales debían completar. Posteriormente, se agendó una cita para entrevista individual con cada participante de la muestra reclutada, la cual fue realizada a través de videoconferencias. En la misma se realizó la entrevista semiestructurada y se verificaron los criterios de inclusión y exclusión. Los participantes seleccionados aprobaron un acuerdo de confidencialidad escrito, enviado en formato digital, con el objetivo de fortalecer las medidas de protección de la privacidad en la realización de intervenciones psicológicas en plataformas digitales.

Se realizaron ocho sesiones de intervención grupal virtual a través de videoconferencias, con una frecuencia de una vez por semana, con una duración de 90 minutos cada sesión. Durante la última sesión, se administraron nuevamente las Escalas de Bien- 


\section{Intervención grupal online para la elaboración del duelo y recuperación del bienestar psicológico \\ tras una ruptura amorosa}

estar Psicológico (Rodríguez-Carvajal et al., 2010) en formato digital y se agregó una pregunta cualitativa con el objetivo de identificar su percepción sobre los aportes del programa a su bienestar psicológico.

El análisis de los resultados cuantitativos se realizó con el programa estadístico JASP Team (2018). El análisis cualitativo se hizo a través de la técnica de análisis de contenido por categorías.

\section{Resultados}

\section{Análisis demográfico}

La muestra final del estudio estuvo compuesta por 12 participantes, con una edad promedio de 29.08 ańos $(D E=10.75)$. En cuanto al nivel académico alcanzado, el $58.33 \%$ se encontraba en nivel universitario, el $25 \%$ en nivel de posgrado y 16.67 \% había alcanzado el nivel secundario. El 83.33\% de los participantes había terminado una relación de noviazgo, mientras que el $16.67 \%$ de los participantes se encontraba en proceso de divorcio.

La media de duración de las relaciones terminadas fue de 43 meses $(D E=47.46)$, siendo la más larga de 144 meses y la más corta de 5 meses. La media del tiempo transcurrido desde la ruptura hasta la intervención fue de 9.58 meses $(D E=15.42)$.

\section{Hallazgos cuantitativos}

En la evaluación pre-test la puntuación media para la muestra final fue de $m=219.00(D E=23.01)$. La tabla 1 muestra las puntuaciones promedio por escala. Se verifica que las escalas con menor puntuación media son la autoaceptación, con una media de $24.25(D E=3.57)$; relaciones positivas, con una media de $24.50(D E=5.87)$; y propósito de vida, con una media de $25.33(D E=3.22)$. Esto pudiera sugerir que, tras haber vivido una ruptura amorosa, estas dimensiones del bienestar psicológico se ven afectadas en los participantes.
Tabla 1. Puntuaciones medias pre-test Escalas de Bienestar Psicológico

\begin{tabular}{|l|c|c|c|c|}
\hline ESCALA & $\boldsymbol{m}$ & $\boldsymbol{D E}$ & MIN & MAX \\
\hline Autoaceptación & 24.25 & 3.57 & 16 & 29 \\
\hline Autonomía & 31.42 & 7.49 & 16 & 42 \\
\hline Relaciones positivas & 24.5 & 5.87 & 15 & 34 \\
\hline Dominio del entorno & 25.67 & 3.8 & 19 & 32 \\
\hline Crecimiento personal & 32.5 & 4.42 & 26 & 41 \\
\hline Propósito de vida & 25.33 & 3.23 & 21 & 30 \\
\hline Vitalidad & 27.5 & 4.44 & 21 & 36 \\
\hline Recursos internos & 27.83 & 4.35 & 18 & 35 \\
\hline TOTAL & $\mathbf{2 1 9}$ & $\mathbf{2 3 . 0 1}$ & $\mathbf{1 9 2}$ & $\mathbf{2 6 7}$ \\
\hline
\end{tabular}

Fuente: Rodríguez-Carvajal et al., 2010.

Luego de la intervención (post-test), la puntuación media global en las Escalas de Bienestar Psicológico (Rodríguez-Carvajal et al., 2010) fue de 247.58 $(D E=21.40)$. La tabla 2 muestra las puntuaciones medias por escala luego de la intervención. Estos resultados evidencian un aumento en los niveles de Bienestar Psicológico de los participantes luego de la intervención.

Tabla 2. Puntuaciones medias post test Escalas de Bienestar Psicológico

\begin{tabular}{|l|c|c|c|c|}
\hline ESCALA & $\boldsymbol{m}$ & $\boldsymbol{D E}$ & MIN & MAX \\
\hline Autoaceptación & 28.75 & 3.55 & 22 & 35 \\
\hline Autonomía & 28.67 & 5.31 & 19 & 35 \\
\hline Relaciones positivas & 35.33 & 3.6 & 31 & 43 \\
\hline Dominio del entorno & 28.25 & 2.49 & 24 & 33 \\
\hline Crecimiento personal & 36.42 & 3.61 & 31 & 42 \\
\hline Propósito de vida & 29.75 & 3.14 & 24 & 35 \\
\hline Vitalidad & 31.75 & 5.68 & 21 & 40 \\
\hline Recursos internos & 28.67 & 3.6 & 24 & 34 \\
\hline TOTAL & $\mathbf{2 4 7 . 5 8}$ & $\mathbf{2 1 . 4}$ & $\mathbf{2 1 1}$ & $\mathbf{2 8 3}$ \\
\hline
\end{tabular}

Fuente: Rodríguez-Carvajal et al., 2010. 
Se llevó a cabo la prueba Shapiro-Wilk para verificar la normalidad de la distribución de las puntuaciones, encontrándose que estas siguen una distribución normal $(p=0.192)$. Asimismo, la $d$ de Cohen (1.69) sugiere que se trata de un efecto importante. No se encontraron valores atípicos.

Se realizó una prueba $t$ de Student de muestras dependientes para verificar si las diferencias en las puntuaciones pre-test y post-test fueron significativas. Se encontraron diferencias significativas en el nivel de bienestar psicológico de los participantes luego de haber realizado el programa de intervención $(p<0.001 ; t=5.86 ; d f=11)$, mostrando un aumento promedio de 28.58 puntos ( $D E=16.91$ ).

Además, se realizaron pruebas de correlación de Pearson y se encontró que no existía relación entre el nivel de bienestar psicológico y la duración de la relación. Tampoco se encontró relación significativa con el tiempo transcurrido desde la ruptura ( $>0.050)$.

\section{Hallazgos cualitativos}

A continuación, se presentan los resultados del análisis cualitativo de contenido. Los datos cualitativos recolectados a través de una entrevista semiestructurada de preguntas abiertas fueron organizados en cinco categorías principales, y cuatro subcategorías.

Categoría 1: Consecuencias en el ámbito emocional. Esta categoría engloba las consecuencias emocionales experimentadas por los participantes a raíz de la ruptura de la relación de pareja.

Subcategoría 1.1: Malestar emocional. Esta subcategoría se refiere a las manifestaciones emocionales vividas luego de la ruptura, que generan malestar. Todos los participantes reportaron consecuencias negativas en su estado de ánimo posterior a la ruptura de la relación de pareja, haciendo referencia a la experimentación de emociones como la tristeza, rabia, ansiedad, soledad, miedo, sensación de vacío, desesperanza, culpa e irritabilidad. También se encontró que para una participante era difícil expresar con palabras las emociones vividas luego de la ruptura.

Al preguntar sobre las consecuencias emocionales de la ruptura, estas fueron algunas de las respuestas de los participantes:

"Los primeros meses yo lloraba todos los días [...] estaba ansiosa de si me lo encontraba en la calle, no estaba cómoda en ningún lado, de verdad me afectó muchísimo... aún me sigue afectando" (P1, 25 años).

"Antes me sentía como devastada pero ahora como un vacío, como ahora mismo están pasando muchas cosas importantes en su vida y yo no puedo estar ahî” (P8, 26 años).

"Estoy triste porque tengo la sensación de que me falta alguien que me acompañaba, que estaba aquí, con quien yo podía hablar. Cada vez que me pasa una pérdida me arropa una desesperanza terrible, y eso lo necesito trabajar, porque esa desesperanza comienza a darme con el látigo de la culpa y comienzo a crearme una serie de culpas que no tengo... también mucha rabia, mucha rabia" (P11, 42 años). "No tengo como palabras para describir todas las emociones que he vivido" (P2, 24 años).

\section{Subcategoría 1.2: Cambios emocionales.} Esta subcategoría hace referencia a los cambios en las emociones sentidas, que los participantes han ido experimentando a lo largo del tiempo. La mayoría reporta haberse sentido mejor con el paso del tiempo, sin embargo, expresaron seguir experimentando malestar de forma momentánea o en niveles más bajos antes de la intervención."Ya es diferente, es como que... no digo que lo he superado, pero sí lo he entendido. Ya no me siento culpable, 
por ejemplo, antes yo me sentía culpable como que era yo, ya no..." (P11, 42 años).

"Yo entiendo que sí (me sigue afectando), no tanto como antes, puedo decir que he mejorado, pero siento que todavía me afecta, cuando me dicen que se lo encuentran en la calle me duele, me afecta”" (P8, 26 años)." "Sí, realmente yo no he sanado completamente porque por ejemplo me molesta verlo, saber de él, me molesta pensar en él, me molesta mucho todavía, como que me duele" (P3, 24 años).

\section{Categoría 2: Impacto en el ámbito cognoscitivo.} Esta categoría engloba las manifestaciones de tipo cognoscitivo del proceso de duelo, reportadas por los participantes.

Subcategoría 2.1: Autoconcepto. Esta subcategoría se refiere al impacto que los participantes perciben tuvo la ruptura amorosa en la manera en su autoconcepto. La mayoría de los participantes refieren que su imagen propia se vio afectada por la ruptura, expresando que les hizo cuestionarse a sí mismos, percibir que habían fallado o hecho algo mal. También se evidencia que, en algunos casos, posiblemente el impacto que tiene esta experiencia en la imagen de sí mismos, se vincula a los esquemas de pensamientos y creencias que tienen los participantes, como los asociados a la importancia de su propia imagen ante los demás, o la atribución de responsabilidad o culpabilidad sobre las cosas que les suceden. Se muestra que incluso algunos que perciben tener una imagen positiva de sí, reportan haber cuestionado esta imagen a partir de la experiencia de ruptura. Así lo evidencian los testimonios presentados a continuación:

"Totalmente, totalmente, yo me sentía... ¿qué yo te puedo decir? Lo más bajo y lo más chiquito que tú te puedas imaginar. Mi autoestima se fue al piso completamente". (P11, 42 años)
"En parte sí, porque yo soy una persona que siempre anda como con mucho cuidado de lo que la gente va a decir de mí, como cuidando mi imagen... entonces la gente ve como que yo no lo satisfago completamente. (He pensado) que si yo estoy haciendo algo mal porque ya van varias relaciones que terminan, ya cuatro... Y me hace como analizar si soy yo la que tiene el error, pero yo sé que yo no soy totalmente culpable de la ruptura de la relación.” (P7, 20 años)

"Sí, bastante, porque me siento muy íntegro, recto, muy todo perfectico, soy muy metódico, todo tiene que estar en su sitio. Entonces, veo esto como un fracaso, algo que fallé... no sé en qué fallé, pero lo veo como que no pude. Sí, me afectó eso mismo, el tema de la integridad, lo que dice la gente tal vez..." (P12, 41 años) "Yo siempre he sido muy seguro de mí... pero en el punto que yo siento que esto me ha afectado más es en que yo siento como que yo no...yo no sé si es que yo no encajo... hay gente que quisiera estar conmigo y yo como que no pego con nadie." (P9, 28 años)

\section{Subcategoría 2.2.: Otras manifestaciones cognos-} citivas. Esta subcategoría engloba otras consecuencias de tipo cognoscitivo que los participantes experimentaron luego de la ruptura de su relación de pareja. Reportan confusión, tener pensamientos constantes sobre la ruptura, la expareja u otros temas asociados, así como problemas de concentración a partir de estos.

"Como que siempre pienso en eso y como que "ella lo hace feliz, era por ella", o sea tengo todas esas dudas como que no me dejan enfocarme en otra cosa. Ahora mismo me la paso pensando qué estará haciendo él... me enteré, lo vi que estaba saliendo con otra gente así tan rápido y como que siempre pienso en eso." (P8, 26 años) 
"Realmente sí, en el trabajo, desde enero yo estoy como ida de la cabeza, haciendo tollos [cometer errores]." (P10, 21 años)

"En parte sí [tenía dificultades en el trabajo], porque me distraía, me iba, me ponía a pensar... me levantaba más tarde, cosas así." (P3, 24 años) "Todavía me siento súper confundida, o sea, de verdad yo todavía no entiendo nada." (P1, 25 ańos)

\section{Categoría 3: Manifestaciones en el ámbito} conductual. Esta categoría engloba las manifestaciones conductuales del duelo vividas por los participantes. La principal manifestación reportada, es la evitación de lugares, actividades e incluso objetos. La mayoría expresa haber evitado o seguir evitando distintas cosas que evocan recuerdos de la expareja o la relación. También algunos participantes reportaron otras manifestaciones conductuales como el llanto, cambios en la alimentación, la búsqueda de la expareja y el aislamiento. Una de las participantes incluso reportó haberse mudado de ciudad temporalmente a raíz de la ruptura de la relación.

"Sí, me fui de la ciudad en la que vivía. Necesitaba la protección de mi familia, apoyo porque sola no iba a poder mantenerme. Salí de mi lugar que me hacía feliz para tener protección y seguridad." (P2, 24 años)

"Bueno, todo lo que yo tengo que ella me ha dado, pijamas, regalos, todas las saqué... cosas que me recuerdan a ella." (P6, 27 años)"Eliminé las conversaciones, eliminé las fotos, saqué todo lo que tenía de él en mi cuarto, eliminé Twitter, porque ya me estaba afectando demasiado." (P7, 20 ańos)

“Teníamos un grupo de la iglesia en común al que yo dejé de asistir porque no tolero verlo todavía." (P3, 24 años)
Categoría 4: Manifestaciones físicas. Algunos participantes reportaron haber experimentado síntomas físicos durante el proceso de duelo tras la ruptura de su relación, en la forma de cambios en el peso y náuseas.

"No estaba comiendo, perdí muchísimas libras al punto de que todos mis pantalones me quedan grandísimos." (P1, 24 años)

"Cada vez que yo me lo imagino con él se me quitan las ganas de comer, eh se me quita el apetito. Si me estoy cepillando y me acuerdo de eso me da una náusea horrible, y cuando empiezo a llorar me da náusea y náusea y náusea." (P7, 20 años)

\section{Categoría 5: Luego de la intervención}

Esta categoría se refiere a los cambios percibidos por los participantes luego de la intervención de ocho semanas. Algunos de los participantes expresaron que luego de la intervención sienten una sensación de alivio, o de haberse quitado un peso de encima. Otros, expresaron percibir una mejoría en su estado emocional, en comparación con lo que sentían antes del programa, destacando un sentimiento de esperanza hacia el futuro. Los siguientes testimonios evidencian estas percepciones:

"Fueron ocho semanas cargadas de muchos sentimientos, pero sobre todo de mucho alivio. Llegué sin poder encontrar el sueño por las noches, buscando palabras de aliento y hoy me voy con mucha paz y reconociendo que todo en la vida pasa." (P2, 24 años)

"Entiendo que he evolucionado y me siento liberado, con la carga sobre mis hombros más liviana y muy motivado a cerrar ese capítulo en mi vida. Al hacer eso, me abro de nuevo a la vida, a conocer personas, a tratar más profundamente a quienes se me han acercado y he rechazado, ya sea por temor a más 
dolor o simplemente en espera de un imposible que hace mucho sé que no va a suceder. Ya no me aferro a esa idea de que volverá y veo hacia el futuro esperanzado de mi nuevo resurgir." (P12, 41 años)

"No es que estoy saltando en un pie de alegría, pero estoy mejor, y sé que voy a estar mejor." (P3, 24 ańos)

Otros participantes expresaron que la intervención grupal les permitió reconocer que no son las únicas personas pasando por una situación dolorosa. El desarrollo de la empatía con los demás participantes es reconocido como un beneficio por ellos y ellas.

"Me encantó aún más ver que no estaba sola, que todos ustedes pasaron por lo mismo o parecido, y que todos reconocemos que debemos ahora ser mucho más fuertes por nosotros mismos y por nadie más." (P8, 26 años)

"Vi en ellos mis mismas lágrimas y dolores y eso me creó empatía. Hoy no los veo como desconocidos, los veo como guerreros en la misma batalla que yo libraba, hoy veo en ellos personas que quieren dar lo mejor de sí para sí mismos, veo en mis compañeros la misma inercia que me lleva a mí a mirar hacia adelante." (P12, 41 años)

"El grupo y escuchar las experiencias de mis compañeros me ayudaron bastante para visualizarme desde otra perspectiva." (P4, 54 ańos)

También a partir de otros testimonios se evidencia un cambio de ideas erróneas que podían dificultar el procesamiento adecuado del duelo, por ejemplo:

"Creía que el duelo por una ruptura era algo de débiles, me doy cuenta de que no. Hay que ser muy fuerte... me doy cuenta de ideas erróneas que tengo y debo trabajar para no repetir patrones." (P1, 25 años)
"Mi fe en la terapia grupal no era muy alta. Esta oportunidad cambió completamente esa creencia para mí." (P5, 26 años)

Además, se evidencia un cambio de actitud hacia la idea de los espacios de intervención grupales. Por tanto, podemos inferir que participar de este programa también pudiera generar cambios positivos en algunas personas con relación a su actitud de recibir asistencia profesional cuando se enfrentan a situaciones difíciles como el duelo.

Algunos participantes también expresaron que luego de la intervención han identificado o reconocido algunos temas que deben ser atendidos en otros espacios terapéuticos, o que aún quedan tareas del duelo por resolver. Expresan sentirse motivados a continuar este proceso. Así lo evidencian los siguientes testimonios:

"Me siento feliz por los resultados obtenidos personalmente, nostálgica de que todavía hay cosas que duelen, pero con ganas de superarlo." (P6, 27 años) "Al concluir, sé que mi proceso no ha terminado, pero que ahora lo enfrento con más armas y comprometida a seguir trabajando para superarme y encontrarme al final de este camino una mejor versión de mí." (P5, 26 años)

\section{Discusión}

Los resultados cualitativos agrupados en las cuatro primeras categorías evidencian que los participantes han experimentado distintas manifestaciones emocionales, cognoscitivas, conductuales y fisiológicas a partir de la ruptura de una relación amorosa significativa para ellos. Entre estas manifestaciones se pueden encontrar la pérdida de peso, el aislamiento, dificultades para la concentración y la experimentación de emociones como la tristeza, la ira, y la culpa. Esto confirma lo planteado por distintos autores en la literatura, quienes identifican estas manifestaciones como comunes durante los procesos de duelo. 
Además, se evidencia que estos signos no son exclusivos de los duelos por muerte de personas significativas, sino que también se experimentan luego de la pérdida de una relación amorosa (Worden, 2018; Sierra et al., 2017; Muñiz Tinajero, 2010 citados por Aragón \& Cruz, 2014; Cuervo et al., 2009; Testor et al., 2009; Morales, 2019; Yárnoz-Yaben, 2017; Raya et al., 2013).

Por otro lado, como se indicó en el análisis sociodemográfico, el $88 \%$ de la muestra había terminado una relación de noviazgo, mientras que solo el $12 \%$ había terminado un matrimonio. El hecho de que todos los participantes, independientemente del tipo de relación que sostenían, reportaran distintas formas de malestar, evidencia lo planteado por Sierra et al. (2017) quienes afirman que, a pesar de que la mayoría de las consecuencias de las rupturas de pareja se han estudiado con relación al divorcio, el impacto de una ruptura también es vivido por las personas que, aun no habiendo formalizado un matrimonio, desarrollaron una relación estable y que consideran seria.

La diversidad de respuestas emocionales, cognitivas, conductuales y fisiológicas reportadas por los participantes del estudio, pudiera ser un indicador de lo planteado en la literatura por distintos autores, que señalan que los síntomas que han sido descritos como comunes en procesos de duelo normal, no son un cuadro fijo. Los hallazgos de este estudio comprueban que durante una ruptura de pareja la experiencia del duelo es vivida por cada persona de manera distinta (Worden, 2018; Yárnoz-Yaben, 2017; Bucio, 2007 citado por Raya et al., 2013; Testor et al., 2009; Fernández- Liria et al., 2006).

Los hallazgos de este estudio evidencian un aumento significativo en el nivel de bienestar psicológico de los participantes luego de haber realizado el programa de intervención $(p<0.001 ; t=5.86$; $d f=11)$, con un promedio de $28.58(D E=16.91)$. Estos resultados pudieran indicar que el programa de intervención diseñado es efectivo para la recuperación del bienestar psicológico luego de haber sufrido una ruptura de pareja. Los hallazgos de este estudio se corresponden con lo planteado por Schut (2010), citado por Worden (2018), quien afirma que cuando algunas personas experimentan dificultades durante su proceso de duelo, y buscan acompañamiento profesional porque perciben que puede ser de ayuda, la intervención puede facilitar una adaptación más efectiva a la pérdida. Además, sustenta lo comprobado en estudios anteriores, en los cuales la realización de intervenciones grupales con personas que están atravesando un proceso de duelo han tenido resultados positivos (Quinney \& Fouts, 2008; Vukalovich \& Caltabiano, 2008; Raya et al., 2013; Najjari et al., 2017; Nameni et al., 2017; Saadati et al., 2017). Los resultados cualitativos también señalan mejorías significativas en el bienestar psicológico de los participantes, además de otros resultados positivos como el cambio de percepción hacia la intervención psicológica como un recurso, y la formación de una red de apoyo que ha mostrado ser uno de los factores de protección para la vivencia de duelos normales y prevención de duelos patológicos (Villacieros, Serrano, Bermejo, Magaña \& Carabias, 2014)

Estos hallazgos, pudieran ser indicadores de que la intervención grupal puede promover la búsqueda de otros espacios de acompañamiento para el abordaje de complicaciones en el proceso de duelo que pudieran presentarse más adelante, o ante otras pérdidas o situaciones difíciles que enfrenten los participantes en el trayecto de sus vidas. Se evidencia que, para algunos de los participantes, recurrir a la asistencia psicológica es ahora un recurso de afrontamiento a situaciones difíciles.

Esta investigación fue llevada a cabo en el período marzo-junio 2020, durante el estado de emergencia sanitaria global provocado por la pandemia COVID-19. Esto implicó la paralización de todos los servicios presenciales y de toda la actividad 
comercial, por la necesidad de un confinamiento obligatorio como medida de mitigación epidemiológica. Dadas estas circunstancias, fue necesario adaptar todos los servicios de salud mental y atención psicosocial, desarrollando intervenciones a distancia, por vía telefónica o internet. Es en este marco que surge la necesidad de diseñar este programa de intervención en modalidad virtual. Los resultados obtenidos en esta investigación demuestran que las intervenciones a través del internet son una alternativa viable y a partir de las cuales se pudieran obtener resultados positivos en el trabajo de duelo por ruptura amorosa. Corrobora lo encontrado en estudios anteriores, en los cuales intervenciones virtuales mostraron ser efectivas para el trabajo de duelo por distintas pérdidas en poblaciones adultas (Fiegelman et al., 2008 citados por Worden, 2018; Elder \& Burke, 2015; Kersting et al., 2013; Kersting et al., 2011; Wagner \& Maercker, 2008; Wagner et al., 2007).

Cabe destacar que la mayoría de los estudios encontrados sobre trabajo de duelo, así como gran parte de las bases teóricas de la descripción del proceso de duelo, se refieren a las pérdidas por fallecimiento. Asimismo, los instrumentos de medición para duelo son específicos a este tipo de pérdida. El presente estudio es una referencia para continuar el desarrollo de esta área de investigación, con la realización de otros estudios dirigidos a demostrar la eficacia de intervenciones para el trabajo de duelo por rupturas o divorcios, en quienes formaban la pareja, ya que la evidencia empírica sobre intervenciones para este tipo de pérdida es escasa en la literatura, o están enfocadas en el trabajo terapéutico de las consecuencias que tienen estos procesos en los hijos e hijas.

\section{Limitaciones del estudio}

Este estudio tiene varias limitaciones que deben ser mencionadas. En primer lugar, por el tamaño de la muestra y el proceso de selección de esta, los hallazgos de esta investigación no pueden ser generalizados a la población dominicana. Además, por no tener un grupo control, no puede afirmarse que los hallazgos obtenidos son resultado de la intervención aplicada, ya que pudiera haber otras variables influyentes en los resultados.

Otro factor importante en la realización de este estudio es la ocurrencia de una situación de emergencia sanitaria a raíz de la pandemia por COVID19. Algunos autores han afirmado que las situaciones de emergencia de salud pública generan consecuencias psicológicas importantes, afectando la salud mental, la seguridad y el sentido de bienestar de los individuos y las comunidades. Asimismo, diversos estudios realizados con la población China, han encontrado que esta situación crítica ha generado consecuencias psicológicas importantes en la población general, afectando la salud mental (Pfefferbaum \& North, 2020; Wang et al., 2020; Qiu et al., 2020). Por tanto, no puede afirmarse que las manifestaciones cognitivas, emocionales, conductuales y físicas sean producidas exclusivamente por la vivencia de la ruptura amorosa. Estas pudieran haber estado agravadas por la situación de emergencia, el confinamiento y distanciamiento social. Sin embargo, aunque la situación de emergencia pudiera haber agravado o provocado los bajos niveles de bienestar psicológico u otros de los signos reportados por los participantes, se evidencian mejorías luego de la intervención.

A pesar de estas limitaciones, gracias a la naturaleza mixta del estudio, esta investigación aporta riqueza de información cualitativa sobre las consecuencias emocionales, cognoscitivas, conductuales y fisiológicas de las personas que atraviesan un duelo por ruptura amorosa, y cómo estas perciben cambios en sus actitudes, estado de ánimo, pensamientos y emociones luego de haber participado de la intervención. Además, los resultados cuantitativos fueron significativos para la muestra. Por tanto, el presente estudio es un punto de partida para el desarrollo de investigaciones futuras en el ámbito de implementación de programas de intervención para el trabajo de duelo por ruptura amorosa en la población dominicana. 


\section{Recomendaciones}

Partiendo de los hallazgos de este estudio, se exhortan investigaciones a modo de réplica, con un diseño de tipo experimental con grupo control, con el propósito de hacer inferencias y generalizaciones a grupos mayores y verificar la eficacia de la intervención, pudiendo establecer relación de causalidad entre la intervención y niveles más elevados de bienestar psicológico. Además, se recomienda hacer estudios con evaluación de seguimiento (follow up), para determinar si los cambios observados luego de la intervención permanecen a lo largo del tiempo.

Diversos autores han explicado que la forma en que se manifiesta el duelo tras una pérdida amorosa depende de diversos factores como la historia personal, la personalidad, el contexto sociocultural, el estilo de apego, las estrategias de afrontamiento, el apoyo social recibido y percibido, la cercanía de la relación que ha terminado y el grado de compromiso. Futuras investigaciones pudieran dirigirse a verificar de qué manera cada uno de estos factores influye en el tipo de síntomas o manifestaciones que presenta la persona, y en cómo transcurre la adaptación que supone vivir un proceso de duelo atendiendo a estas variables (Worden, 2018; Yárnoz-Yaben, 2017; Bucio, 2007 citado por Raya et al., 2013; Testor et al., 2009; Fernández-Liria et al., 2006).

\section{Referencias}

Aragón, R.S. \& Cruz, R.M. (2014) Causas y caracterización de las etapas del duelo romántico. Acta de Investigación Psicológica, 4(1), 1329-1343. Recuperado de: https://doi. org/10.1016/S2007-4719(14)70378-3

Cuervo, Á., Moreno, G., \& Concha, E. (2009). Percepciones de mujeres divorciadas acerca del divorcio. Enseñanza e investigación en psicologia, 14(1), 23-35. Recuperado de: https:// www.redalyc.org/articulo.oa?id=29214102
Elder, J., \& Burke, L. A. (2015). Parental grief expression in online cancer support groups. Illness, Crisis y Loss, 23(2), 175-190. Recuperado de: https://doi.org/10.1177/1054137315576617

Fernández-Liria, A., Rodríguez-Vega, B. \& Diéguez-Porres, M. (2006) Intervenciones sobre duelo. En P. Pérez-Sales, (ed.). Trauma, culpa y duelo: Hacia una psicoterapia integradora. Bilbao: Desclée de Brouwer. Recuperado de: http://afliria.info/download/inter venciones-sobre-duelo/

Guillén, E. G., Montaño, M. J. G., \& Gordillo, M. D. G. (2015). El grupo me ayudó a no estancarme. el avance en el proceso de duelo. International Journal of Developmental and Educational Psychology, 2(1), 469-476. Recuperado de: https://www.redalyc.org/articulo. oa?id=349851784046

Kersting, A., Dölemeyer, R., Steinig, J., Walter, F., Kroker, K., Baust, K., \& Wagner, B. (2013). Brief Internet-based intervention reduces posttraumatic stress and prolonged grief in parents after the loss of a child during pregnancy: a randomized controlled trial. Psychotherapy and Psychosomatics, 82(6), 372-381. Recuperado de: https://doi.org/10.1159/000348713

Kersting, A., Kroker, K., Schlicht, S., Baust, K., \& Wagner, B. (2011). Efficacy of cognitive behavioral internet-based therapy in parents after the loss of a child during pregnancy: pilot data from a randomized controlled trial. Archives of women's mental health, 14(6), 465-477. Recuperado de: https://link. springer.com/article/10.1007/s00737-0110240-4

Lee, J. \& Hett, G. (1990). Post-divorce Adjustment: An Assessment of a Group Intervention. Canadian Journal of Counselling and Psychotherapy, 24(3), 199-209. Recuperado de: https://cjc-rcc.ucalgary.ca/article/view/59513 Morales, J. (2019). Proceso de duelo por ruptura de pareja. Ingenio Social, 8(8), 46-67. Recuperado de: http://pluseconomia.unachi.ac.pa/ index.php/ingeniosocial/article/view/362 
Najjari, F., Khodabakhshi Koolaee, A., \& Falsafinejad, M. (2017). The effectiveness of group therapy based on Acceptance and Commitment (ACT) on loneliness and psychological adjustment in women after divorce. Journal of Torbat Heydariyeh University of Medical Sciences, 5(3), 68-75. Recuperado de: http:// jms.thums.ac.ir/browse.php?a_code=A-10461-2\&sid=1\&slc_lang=en

Nameni, E., Mohammadipoor, M., \& Noori, J. (2017). The effectiveness of emotion-focused group therapy on interpersonal forgiveness and hope in divorced women. Counseling Culture and Psychotherapy, 8(29), 57-78. Recuperado de: https://www.sid.ir/en/journal/ViewPaper. aspx?id $=606335$

Pfefferbaum, B. \& North, C. (2020). Mental Health and de COVID19 Pandemic. New England Journal of Medicine. Recuperado de: https://www.nejm.org/doi/full/10.1056/ NEJMp2008017

Qiu J., Shen B., Zhao M., Wang Z., Xie B., Xu Y. (2020). A nationwide survey of psychological distress among Chinese people in the COVID-19 epidemic: implications and policy recommendations. General Psychiatry, 33(2): e100213. Recuperado de: http://dx.doi. org/10.1136/gpsych-2020-100213

Quinney, D. \& Fouts, G. (2008) Resilience and Divorce Adjustment in Adults Participating in Divorce Recovery Workshops. Journal of Divorce \& Remarriage, 40(1-2), 55-68. Recuperado de: https://doi.org/10.1300/J087v40 n01_04

Raya, A. R., Sierra, M. I. M., \& Velasco, N. O. (2013). Psicoeducación para el proceso de duelo después del divorcio en Morelia, Michoacán. Aportes Interdisciplinarios en el Ejercicio Profesional de la Salud Mental, 2, 127-138. Recuperado de: https://scholar. googleusercontent.com/scholar?q=cache: $4 \mathrm{Yy}$ mWNyMpJcJ:scholar.google.com/+Raya,+A.+R.,+Sierra,+M.+I.+M.,+\%26+Velasco, + N.+O.+(2013) .+Psicoeducaci\% -
$\mathrm{C} 3 \% \mathrm{~B} 3 \mathrm{n}+$ para + el + proceso $+\mathrm{de}+\mathrm{duelo}+-$ despu\%C3\%A9s+del+divorcio+en+Morelia,+Michoac\%C3\%A1n\&hl=es\&as_sdt=0,5

Rodríguez-Carvajal, R., Díaz, D., Moreno-Jiménez, B., Blanco, A. \& Van Dierendonck, D. (2010). Vitalidad y Recursos Internos como Componentes del Constructo de Bienestar Psicológico. Psicothema, 22(1), 63-70. Recuperado de: http://www.psicothema.com/ english/psicothema.asp?id=3697

Saadati, N., Rostami, M., \& Darbani, S. (2017). Comparing the effectiveness of Acceptance and Commitment Therapy (ACT) and Compassion Focused Therapy (CFT) on improving self-esteem and post-divorce adaptation in women. Family Psychology, 3(2), 45-58. Recuperado de: https://www.sid.ir/ En/Journal/ViewPaper.aspx?ID=542984

Sampieri, R., Collado, C., \& Lucio, M. (2010). Metodología de la Investigación. México: McGraw-Hill.

Sierra, V., Rodríguez, J., \& Torres-Torija, C. (2017). Incidencia del duelo en la ruptura amorosa en estudiantes universitarios en un Centro de Crisis, Emergencias y Atención al Suicidio (CREAS). Journal of Behavior, Health y Social Issues, 9(2), 27-35. Recuperado de: https:// doi.org/10.1016/j.jbhsi.2018.01.001

Testor, C., Pujol, M., Vidal, C. \& Alegret, I. (2009) El divorcio: una aproximación. La Revue du REDIF, 2, 39-46. ISSN: 2070-9021. Recuperado de: https://www.researchgate.net/profile/ Carles_Testor/publication/242775375_El_ divorcio_una_aproximacion_psicological links/54e43ae50cf282dbed6ea7ba/El-divorcio-una-aproximacion-psicologica.pdf

Villacieros, M., Serrano, I., Bermejo, J. C., Magaña, M., \& Carabias, R. (2014). Social support and psychological well-being as possible complicated grief level predictor in a grief population sample. Anales de Psicología, 30(3), 944-951. Recuperado de: https://doi.org/10.6018/ analesps.30.3.154691 
Vukalovich, D. \& Caltabiano, N. (2008) The Effectiveness of a Community Group Intervention Program on Adjustment to Separation and Divorce. Journal of Divorce y Remarriage, 48 (3-4), 145-168. Doi: 10.1300/J087v48n03_09

Wagner, B., \& Maercker, A. (2008). An Internet-based cognitive behavioral intervention for complicated grief: A pilot study. Giornale italiano di medicina del lavoro ed ergonomia, 30(SupplB), B47-B53. Recuperadode: https:// www.researchgate.net/profile/Birgit_Wagner/ publication/24203193_An_internet-based_ cognitive-behavioral_preventive_intervention_for_complicated_grief_A_pilot_study/ links/0deec525fb1a04305c000000/ An-internet-based-cognitive-behavioral-preventive-intervention-for-complicated-grief-A-pilot-study.pdf

Wagner, B., Knaevelsrud, C., \& Maercker, A. (2007). Post-traumatic growth and optimism as outcomes of an internet-based intervention for complicated grief. Cognitive behaviour therapy, 36(3), 156-161. Recuperado de: https://doi. org/10.1080/16506070701339713
Wang, C.; Pan, R.; Wan, X.; Tan, Y.; Xu, L.; Ho, C.S.; Ho, R.C. (2020). Immediate Psychological Responses and Associated Factors during the Initial Stage of the 2019 Coronavirus Disease (COVID-19) Epidemic among the General Population in China. Int. J. Environ. Res. Public Health 2020, 17, 1729. Recuperado de: https://doi.org/10.3390/ ijerph17051729

Worden, J. W. (2018). Grief Counselling and Grief Therapy: A Handbook for the Mental Health Practitioner $\left(5^{\text {th }}\right.$ ed.). New York: Springer Publishing Company.

Yárnoz-Yaben, S. (2017). El duelo ante el divorcio: relación con el estilo de apego y efectos en el bienestar subjetivo y el ejercicio de la coparentalidad. Studies in Psychology, 38(3), 667-688, Doi: 10.1080/02109395.2017.1330305.

Yárnoz-Yaben, S., \& González, P. C. (2012). Un instrumento para la evaluación del perdón en el ámbito del divorcio y la separación. International Journal of Psychology and Psychological Therapy, 12(1), 49-58. Recuperado de: https:// www.redalyc.org/pdf/560/56023335004.pdf

\section{Datos de filiación}

Perla Navarro Vásquez. Licenciada en Psicología por el Instituto Tecnológico de Santo Domingo (INTEC). Especialidad en intervenciones en crisis y trauma otorgada por la Universidad Iberoamericana (UNIBE) y la Universidad de Salamanca. Sus líneas de investigación son psicología clínica, psicología social y salud mental. 